\title{
Between Scylla and Charybdis: antipsychotic and other psychotropic medications in older nursing home residents
}

\author{
Bruce G. Pollock MD PhD, Benoit H. Mulsant MD MS
}

See related research article by Huybrechts and colleagues at www.cmaj.ca/cgi/doi/10.1503/cmaj.101406.

Competing interests:

Bruce Pollock has served as an advisory board member for Lundbeck Canada, received compensation from the Lundbeck International Neuroscience Foundation for faculty member expenses, and consulted for Wyeth and for Takeda. Benoit Mulsant has received donations of medication from Bristol-Meyers Squibb, Eli Lilly, Pfizer and Wyeth for clinical trials funded by the US National Institute of Health, and has owned stocks (sold in 2009) in AkzoNobel, Alkermes, AstraZeneca, Biogen Idec, Celsion, Elan, Eli Lilly, Forest, Orchestra

Therapeutics and Pfizer.

This article was solicited and has not been peer reviewed.

Correspondence to: Dr. Bruce G. Pollock, bruce.pollock@utoronto.ca

CMAJ 2011. DOI:10.1503 /cmaj.110348

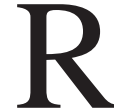
estlessness, disruptive vocalizations and physical aggression are among the most distressing manifestations of dementia and may occur in up to $90 \%$ of patients with dementia at some time during their illness. ${ }^{1}$ These symptoms (often referred to as behavioural and psychological symptoms of dementia), are associated with additional disability, increased burden to caregivers and premature admission to longterm care facilities. ${ }^{1}$ The optimal management of dementia symptoms includes the search for and treatment of physical and environmental precipitants. Nevertheless, symptomatic treatment with a psychotropic medication is frequently required. ${ }^{1}$

Currently, risperidone is the only medication approved in Canada for short-term symptomatic management of inappropriate behaviour owing to aggression or psychosis of severe dementia. Both risperidone and olanzapine were associated with modest improvements in behavioural and psychological symptoms of dementia in the Clinical Antipsychotic Trials of Intervention Effectiveness in patients with Alzheimer's Disease (CATIE-AD) study funded by the United States National Institute of Health (NIH). ${ }^{2}$ However, these medications were also associated with early discontinuations because of adverse effects in a significant proportion of patients. In a metaanalysis of 16 placebo-controlled trials of atypical antipsychotics for behavioural and psychological symptoms of dementia (aripiprazole [3 studies], olanzapine [5 studies], quetiapine [3 studies] and risperidone [5 studies]), death was found to occur more often among patients randomized to medication than among those ran-

\footnotetext{
- KEY POINTS

- Psychotropic medications or the conditions for which they are prescribed may increase the risk of death in older nursing home residents.

- The risk-benefit ratio of any medication must be carefully evaluated on an individual basis for older patients.

- There is an urgent need for evidence-based pharmacologic and nonpharmacologic treatments for the behavioral and psychological symptoms of dementia.
}

domized to placebo (118 [3.5\%] v. 40 [2.3\%]). ${ }^{3}$ Since 2005, "black-box" warnings from the US Food and Drug Administration and advisories from Health Canada caution about this risk of increased mortality associated with use of atypical antipsychotics in older persons with dementia.

In this issue of CMAJ, Huybrechts and coauthors ${ }^{4}$ report that initiation of a conventional antipsychotic, an antidepressant or a benzodiazepine after admission to a nursing home is associated with risks of death or hospitalization as high or higher than after initiation of an atypical antipsychotic. They also report that the risks associated with initiation of a psychotropic medication appear to be similar in patients with and without a diagnosis of dementia.

Several large pharmacoepidemiologic studies (e.g., Kales and colleagues, ${ }^{5}$ Wang and colleagues ${ }^{6}$ ) have shown that the risk of death or fracture associated with use of conventional antipsychotics in older persons is similar or higher than the risk associated with atypical antipsychotics. The finding that benzodiazepines are associated with comparable risks for mortality and morbidity is not surprising; these medications can induce a vicious cycle of increased sedation, cognitive impairment or even frank delirium, leading to increasing agitation and, in turn, to increased doses of benzodiazepines. ${ }^{7}$

The finding that antidepressants are associated with a similar mortality risk is more surprising. In a large cohort study, older outpatients with dementia in whom an antidepressant was initiated had a risk of death about half as high as those treated with an antipsychotic (and about one-third lower than those who were not receiving any psychotropic medications). ${ }^{5}$ Huybrechts and coauthors point out that, "in the absence of randomized controlled trials, pharmacoepidemiologic studies using large databases are the best option available for defining the comparative safety of the psychopharmacologic treatment regimens used to manage behavioral symptoms in older adults with illnesses causing dementia." However, this approach has its limitations. Based on a number of pharmacoepidemiologic studies, conjugated estrogen was

All editorial matter in CMAJ represents the opinions of the authors and not necessarily those of the Canadian Medical Association. 
believed to decrease heart disease and stroke in postmenopausal women. Only when a large randomized controlled trial was finally conducted was it found that these findings were confounded by the social class of estrogen users. ${ }^{8}$ Huybrechts and colleagues remind us that nonrandomized cohort studies attempt to adjust for, but cannot completely eliminate, confounding biases: the reason for the initiation of antidepressants, rather than the antidepressants themselves, may have increased mortality in the nursing home patients in their study. ${ }^{9}$

Huybrechts and coauthors also did not distinguish among the various antidepressants that were prescribed. Tricyclic antidepressants are potent anticholinergic and class I antiarrhythmic agents, and they have previously been associated with increased mortality among older patients with heart disease. ${ }^{10}$ There is general consensus that, regardless of dose, tertiary tricylics like amitriptyline are inappropriate medications for elderly patients. ${ }^{10}$ Serotonin-norepinephrine reuptake inhibitors may also be associated with substantial cardiovascular risks. ${ }^{11}$ Selective serotonin reuptake inhibitors are considered to be considerably safer in these aspects. ${ }^{12}$

We are not optimistic that large placebocontrolled studies of psychotropic medications involving older nursing home residents will be conducted. We can confirm the opinion of Huybrechts and coauthors that there is very little interest on the part of pharmaceutical companies to conduct trials comparing medications from different classes. In 2002, neither the manufacturer of risperidone nor the manufacturer of citalopram was interested in supporting a comparative placebo-controlled trial involving treatment of behavioural and psychological symptoms of dementia. When we submitted a proposal to the $\mathrm{NIH}$ to conduct such a trial, reviewers commented that inclusion of a placebo arm would be unethical given that the efficacy of risperidone had been established. We eventually received funding to conduct a comparative study without a placebo arm. ${ }^{13}$ Rates of completion did not differ between the two drugs (overall completion rate $44 \%$ ), and agitation and psychosis declined similarly in both treatment groups. Although we found no statistical difference, we would be the first to say that the absence of a placebo arm made the results of our study hard to interpret. Also, practice should certainly not be altered on the basis of one study. Consistent with the findings of Huybrechts and coauthors, we would also be concerned that even large clinical trials are unlikely to uncover safety concerns related to rare adverse events, such as hyponatremia, gastrointestinal bleeding or death.

Given the persisting concerns about the safety of antipsychotics and the absence of evidence supporting the efficacy or safety of psychotropic drugs from other classes, clinicians need to consider the risk-benefit ratio for individual patients before initiating pharmacotherapy for behavioural and psychological symptoms of dementia. Clinicians should identify clear target symptoms (e.g., episodes of physical aggression v. wandering, which should not be treated pharmacologically). They should use the lowest possible dose for the shortest possible time, carefully monitor changes in target symptoms and monitor for onset of potential adverse effects. They also need to appreciate that nonpharmacologic behavioural and environmental interventions have an important role in reducing behavioural and psychological symptoms of dementia. However, to be successfully implemented, these interventions require trained staff and resources.

\section{References}

1. Ballard C, Corbett A, Chitramohan R, et al. Management of agitation and aggression associated with Alzheimer's disease: controversies and possible solutions. Curr Opin Psychiatry 2009;22:532-40.

2. Schneider LS, Tariot PN, Dagerman KS, et al. Effectiveness of atypical antipsychotic drugs in patients with Alzheimer's disease. N Engl J Med 2006;355:1525-38.

3. Schneider LS, Dagerman KS, Insel P. Risk of death with atypical antipsychotic drug treatment for dementia: meta-analysis of randomized placebo-controlled trials. JAMA 2005;294:1934-43.

4. Huybrechts KF, Rothman KJ, Silliman RA, et al. Risk of death and hospital admission for major medical events after initiation of psychotropic medications in older adults admitted to nursing homes. CMAJ 2011;183:E411-9.

5. Kales HC, Valenstein M, Kim HM, et al. Mortality risk in patients with dementia treated with antipsychotics versus other psychiatric medications. Am J Psychiatry 2007;164:1568-76.

6. Wang PS, Schneeweiss S, Avorn J, et al. Risk of death in elderly users of conventional vs. atypical antipsychotic medications. $N$ Engl J Med 2005;353:2335-41.

7. Clegg A, Young JB. Which medications to avoid in people at risk of delirium: a systematic review. Age Ageing 2011;40:23-9.

8. Anderson GL, Limacher M, Assaf AR, et al. Effects of conjugated equine estrogen in postmenopausal women with hysterectomy: the Women's Health Initiative randomized controlled trial. JAMA 2004;291:1701-12.

9. Ganguli M, Dodge $\mathrm{HH}$, Mulsant BH. Rates and predictors of mortality in an aging, rural, community based cohort: the role of depression. Arch Gen Psychiatry 2002;59:1046-52.

10. Fick DM, Cooper JW, Wade WE, et al. Updating the Beers criteria for potentially inappropriate medication use in older adults: results of a US consensus panel of experts. Arch Intern Med 2003; 163:2716-24.

11. Johnson EM, Whyte E, Mulsant BH, et al. Cardiovascular changes associated with venlafaxine in the treatment of late-life depression. Am J Geriatr Psychiatry 2006;14:796-802.

12. Lotrich FE, Pollock BG. Aging and clinical pharmacology: implications for antidepressants. J Clin Pharmacol 2005;45:1106-22.

13. Pollock BG, Mulsant BH, Rosen J, et al. A double-blind comparison of citalopram and risperidone for the treatment of behavioral and psychotic symptoms associated with dementia. Am J Geriatr Psychiatry 2007;15:942-52.

Affiliations: Dr. Pollock is with the Division of Geriatric Psychiatry, Department of Psychiatry, University of Toronto, and the Department of Research, Centre for Addiction and Mental Health (CAMH), Toronto, Ontario. Dr. Mulsant is with the Department of Psychiatry, University of Toronto, and the Geriatric Mental Health Program, CAMH, Toronto, Ontario.

Contributors: Both of the authors contributed equally to the drafting and revision of the commentary and approved the final version submitted for publication. 